\title{
ADJUSTING CATCH CURVES FOR GILL NET SELECTION WITH THE LOGISTIC DISTRIBUTION
}

\author{
A.L. JENSEN \\ School of Natural Resources, University of Michigan, Ann Arbor, MI 48109 (U.S.A.)
}

(Accepted 25 January 1982)

\begin{abstract}
Jensen, A.L., 1982. Adjusting catch curves for gill net selection with the logistic distribution. Fish. Res., 1: 155-162.

The normal distribution often is used to describe gill net selection but the normal distribution cannot be integrated in closed form. The logistic distribution, which is a probability distribution similar in shape to the normal distribution, has a probability density function which can be integrated in closed form so that a term for gear selection can be included in the equation describing population size as a function of age. The logistic distribution was applied to the catch curve of Lake Michigan chubs (Coregonus spp.) to adjust it for gill net selection. Yield per 1000 recruits as a function of mean selection age and of fishing mortality was calculated from the adjusted catch curve; these results were compared with yields calculated by conventional methods.
\end{abstract}

\section{INTRODUCTION}

Gear selectivity in fisheries exploited with gill nets often has been described by the normal distribution (Gulland, 1969). Knife-edge recruitment is assumed and individuals of a size between the two inflection points of the normal curve are assumed fully recruited (Gulland, 1969). Knife-edge recruitment describes neither the recruitment process nor gear selectivity accurately, and this method may give results that are difficult to interpret and apply. Since neither recruitment nor selection operates according to the knife-edge concept, methods based on that concept may give erroneous results. Insofar as a catch curve is assumed to correspond to the survival curve of the population from which the catches were taken, it is desirable to find a method by which to adjust a catch curve to eliminate those departures from true survival values that are caused by selection; such a method is available from the use of the logistic distribution.

The logistic distribution is similar in shape to the normal distribution but the density function of the logistic distribution can be integrated in closed form. In analy tical studies the logistic distribution often is used in place of the normal distribution (Johnson and Koltz, 1970). Jensen (1981) used the 
sigmoid-shaped logistic cumulative probability density function to describe trawl selection. In the present study the bell-shaped logistic probability density function is applied to adjust the Lake Michigan chub (Coregonus spp.) catch curve for gill net selection.

The chub fishery is a small but important fishery on the North American Great Lakes. For the conventional stock assessment method yield per 1000 recruits is examined as a function of average fishing mortality and age at entry. For the catch curve adjusted with the logistic selection equation yield per 1000 recruits is examined as a function of the fishing mortality of recruited individuals and mean selection age.

\section{DEVELOPMENT OF THE MODEL}

The bell-shaped logistic probability density function for a random variable $x$ is given by the equation

$p(x)=\{\exp [(x-k) / a]\} / a\{1+\exp [(x-k) / a]\}^{2}$

where $k$ and $a$ are parameters. The mean and variance of the logistic distribution are $E(x)=k$ and $V(x)=a^{2} \pi^{2} / 3$ (Johnson and Koltz, 1970).

Gear selection is a function of size which is most often expressed in terms of length, but length can be transformed to age using the equation (Beverton and Holt, 1957)

$x=x_{0}-\frac{1}{K} \log _{\mathrm{e}}\left[\frac{1-L(x)}{L_{\infty}}\right]$

where $K$ is the growth coefficient, $L_{\infty}$ is asymptotic length, $x_{0}$ is the theoretical age when length equals zero, and $L(x)$ is length at age $x$. The same distribution applied for selection by length can be applied for selection by age (Jensen. 1981).

The yield equation adjusted for gill net selection is similar to the yield equation adjusted for trawl selection developed by Jensen (1981). If fishing mortality and natural mortality remain constant for individuals recruited into the exploited stock, then for the recruited stock the fishing mortality coefficient, $F$, is the product of a catchability coefficient, $q$, and fishing effort, $E$. The yield equation for the recruited stock is $\mathrm{d} Y / \mathrm{d} x=F N(x) W(x)$, where $Y$ is yield and $W(x)$ is the weight of an individual of age $x$. Gear selection causes the catchability coefficient to change with age and thus the fishing mortality coefficient also changes.

The proportion of individuals of age $x$ that become vulnerable at age $x$ is $\phi p(x)$ where $\phi$ is a parameter that adjusts $p(x)$ to one at the age when the cohort is recruited fully. The catchability coefficient can be written as the product of the proportion of the stock vulnerable at age $x, \phi p(x)$, and the catchability coefficient for the recruited stock, $q$; then $\phi p(x) q E=\phi p(x) F$. The yield and mortality equations are

$\mathrm{d} Y / \mathrm{d} x=\phi p(x) F N(x) W(x)$ 
$\mathrm{d} N / \mathrm{d} x=-(\phi p(x) F+M) N(x)$

where $M$ is the natural mortality coefficient. Integration of equation (4) under the initial condition $N(x)=N(k)$ when $x=k$ gives the adjusted catch curve

$N(x)=N(k) \exp \left[\frac{-\phi F}{2}+\phi F\left(1+\mathrm{e}^{(x-k) / a}\right)^{-1}-M(x-k)\right]$

where $N(k)$ is the number of individuals at the mean selection age, $k$.

To compare yield per recruit using the adjusted catch curve with yield per recruit using the unadjusted catch curve the conventional yield model

$\mathrm{d} Y / \mathrm{d} x=\bar{F} N(x) W(x)$

was applied for the unadjusted curve where $\bar{F}$ was the average fishing mortality coefficient for the recruited stock. The exponential mortality model was used, i.e.,

$\mathrm{d} N / \mathrm{d} x=-M N(x), \quad x_{\mathrm{r}}<x<x_{\mathrm{c}}$
$\mathrm{d} N / \mathrm{d} x=-(\bar{F}+M) N(x), \quad x \geqslant x_{\mathrm{c}}$

where $x_{\mathrm{r}}$ is the minimum age at which the stock could be exploited and $x_{\mathrm{c}}$ is the actual age at which the stock becomes vulnerable. Knife-edge recruitment was assumed as in Gulland (1969).

Weight, $W(x)$, for both approaches was modelled using Von Bertalanffy's equation

$W(x)=W_{\infty}\left(1-\mathrm{e}^{-K\left(x-x_{0}\right)}\right)^{b}$

In equation (9) $W_{\infty}$ is the asymptotic weight, $K$ is a growth parameter, $x_{0}$ is the theoretical age when length equals zero, and $b$ is a constant.

For the adjusted catch curve, yield per 1000 recruits was calculated by numerical integration of the yield equation using the trapezoidal rule with an interval size of 0.25 years. For each interval $W(x)$ was calculated from equation (9), $N(x)$ from equation (5), and $p(x)$ from equation (1).

\section{APPLICATION TO THE CHUB FISHERY}

Seven species of deepwater Coregonus from the Great Lakes have been marketed as chubs during the past century. The bloater (Coregonus hoyi) is the smallest and it is apparently the only species remaining in Lake Michigan. Chubs were not an important commercial species while lake trout (Salvelinus namaycush) were available, and the chub population of Lake Michigan was high until 1955. During subsequent years the lake trout stocks declined as a result of sea lamprey (Petromyzon marinus) predation and overfishing (Jensen, 1978) and the chub stocks declined as fishing effort and yields increased. The maximum catch of $5400000 \mathrm{~kg}$ of chubs was made in 1960. In 1976 a lake-wide ban on chub fishing was necessary to save the remnants of the stock.

Chubs were fished commercially with $2 \frac{1}{4}$ to $2 \frac{1}{2}$ inch $(5.72$ to $6.35 \mathrm{~cm})$ mesh 
monofilament gill nets, and there also was some trawling for smaller chubs from 1959 to 1976. After closure of the fishery in 1976 the stocks were monitored by commercial fishermen catching small quotas using commercial gear and standard gangs of graded mesh gill nets. The stocks also were monitored by experimental trawling.

The estimates of some parameters and the data necessary for estimation of the remaining parameters are summarized by Brown (1979). He found a good fit of the normal distribution to the gill net selection data. The normal curve parameters were estimated as $\hat{\mu}=9$ years and $\hat{\sigma}=2.89$ years. The logistic distribution parameters were obtained from the parameters of the normal distribution as $\hat{k}=\hat{\mu}=9$ and $\hat{a}=\sqrt{ } 3 \hat{\sigma}^{2} / \pi^{2}=1.59$. To fit the logistic distribution to selection data the normal distribution can be fitted using conventional methods described by Holt (1963), Gulland (1969), and Regier and Robson (1966), then these estimates can be used to calculate estimates for the parameters of the logistic distribution.

Brown (1979) applied the regression of total mortality on fishing effort to estimate the natural mortality coefficient as $M=0.54$. Assuming a constant natural mortality coefficient, age-specific fishing mortality coefficients, $F$, were estimated (Brown, 1979). The value of $F$ at age 9 was 1.11. Non-linear least squares was applied to growth data given by Brown (1979) to estimate $K$ as 0.32. Applying the length-weight equation calculated by Brown (1979) gives $W_{\infty}=414 \mathrm{~g}$ and $b=3.39$. It was assumed that $x_{0}=0$. The parameter $\phi$ was estimated as $1 / p(k)=6.25$. A recruitment of 1000 age 1 fish was used for both models. The oldest age attainable was set at 15 years.

The fitted normal and logistic probability density functions are similar (Fig. 1). The logistic distribution has a somewhat higher peak and narrower spread; the means of the selection curves are both age 9 and few fish attain this age in the exploited population (Fig. 2). Vulnerability to the gear in-
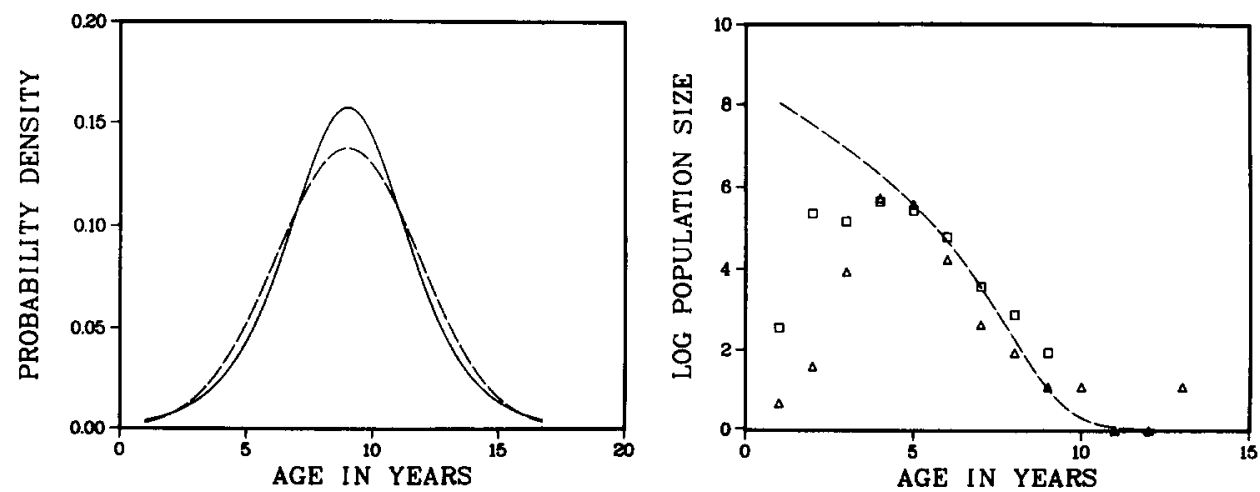

Fig. 1. Comparison of the normal distribution (solid line) and the logistic distribution probability density functions.

Fig. 2. Observed commercial gill net catch curve (triangles), experimental trawl catch curve (squares), and adjusted catch curve. The catch data were not applied for parameter estimation. 
creases continuously over all age groups that are of significant abundance.

Observed gill net and trawl net catch data are plotted with the adjusted catch curve in Fig. 2. These catch data were not applied for parameter estimation but the adjusted catch curve was calibrated so that it gave the same number of individuals of age 5 as were caught with gill nets. The adjusted catch curve fits the data well and enables description of the entire vulnerable lifespan of a cohort. The accuracy of estimates for ages 1,2,3, and 4 cannot be determined, but Jensen (1981) applied the logistic distribution function to describe trawl selection for plaice (Pleuronectes platessa) and showed that the estimates of relative abundance for younger age groups, not yet fully recruited, were comparable to those of a cohort analysis.

Yield per 1000 recruits was calculated using the conventional yield equation and the yield equation based on the adjusted catch curve. The results for the conventional analysis are not directly comparable with the results for the adjusted catch curve because for the conventional approach the fishing mortality coefficient, $\bar{F}$, is an average value for all ages in the exploited stock, whereas for the adjusted curve the fishing mortality coefficient, $F$, is the mortality coefficient for only fully recruited individuals.

First, yield per 1000 recruits was calculated as a function of fishing mortality. To examine the effect of change in $\bar{F}$ with the conventional yield equation the age at entry was set at 5 . To maintain 1000 recruits at age 1 as the fishing mortality coefficient at age $k$ is increased using the adjusted catch curve, the number of individuals of age $k, N(k)$, must be decreased as $F$ is increased. To obtain the necessary values of $N(k)$, equation (5) was solved to give $N(k)$ as a function of $F$ with $N(1)=1000$. The yield per 1000 recruits curve as a function of $\bar{F}$, calculated with the unadjusted catch curve (Fig. 3), is similar in shape to the yield per 1000 recruits curve as a function of $F$ calculated with the adjusted catch curve (Fig. 4); both approach an asymptote. The yield curve
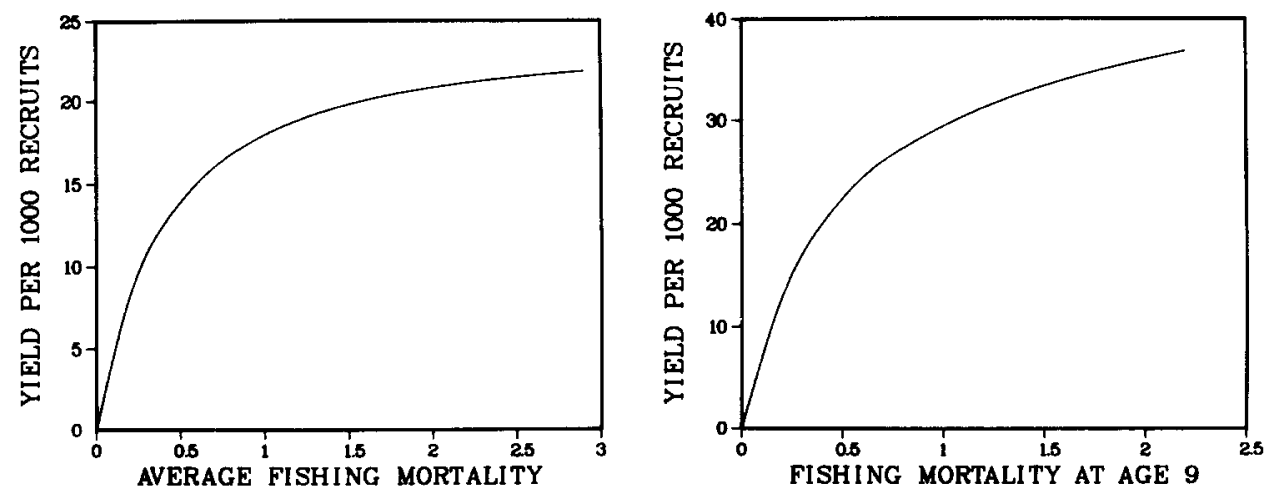

Fig. 3. Yield per 1000 recruits as a function of the average instantaneous fishing mortality coefficient, $\bar{F}$, for the unadjusted catch curve.

Fig. 4. Yield per 1000 recruits as a function of the fishing mortality coefficient, $F$, of fully recruited individuals using the adjusted catch curve. 
calculated using the adjusted catch curve is higher because a considerable portion of the catch is individuals of ages $1,2,3$, and 4 .

An asymptotic relation between yield and fishing mortality occurs when natural mortality is high (Beverton and Holt, 1957). To further explore the two models the curves for yield per recruit as functions of $F$ and $\bar{F}$ were calculated with a lower natural mortality of $M=0.10$. In this case both curves have a maximum at $F=\bar{F}=0.60$ but the two curves are of considerably different shape. The yield curve calculated from the adjusted catch curve has a higher maximum and the peak is more pronounced with a rapidly descending right limb. With the adjusted catch curve younger fish are exposed to fishing mortality, and overfishing these younger rapidly growing fish has a larger impact on yield than overfishing older fish.

To calculate yield per 1000 recruits as a function of the mean selection age, $k$, the number of individuals of age $k$ must be adjusted to maintain 1000 individuals of age 1 . The necessary relation between $k$ and $N(k)$ is given by the adjusted catch curve. For the conventional analysis, to calculate yield per recruit as a function of age at entry, $\bar{F}$ was set at 0.30 . The shape of the curve for yield per 1000 recruits as a function of $k$ (Fig. 6) is considerably different from the shape of the curve for yield per 1000 recruits as a function of $x_{c}$ (Fig. 5). The yield curve calculated using the adjusted catch curve is higher both because more age groups are vulnerable and because the average fishing mortality is higher. For the adjusted catch curve the optimum mean selection age is about 4.5 and with a standard deviation of 2.89 the lower inflection point occurs at about 1.5. The optimum age at entry with the adjusted catch curve is about 1.5, and so the two approaches agree. However, the yield curve calculated with the adjusted catch curve would be more useful for study of the effects of mesh size on yield.
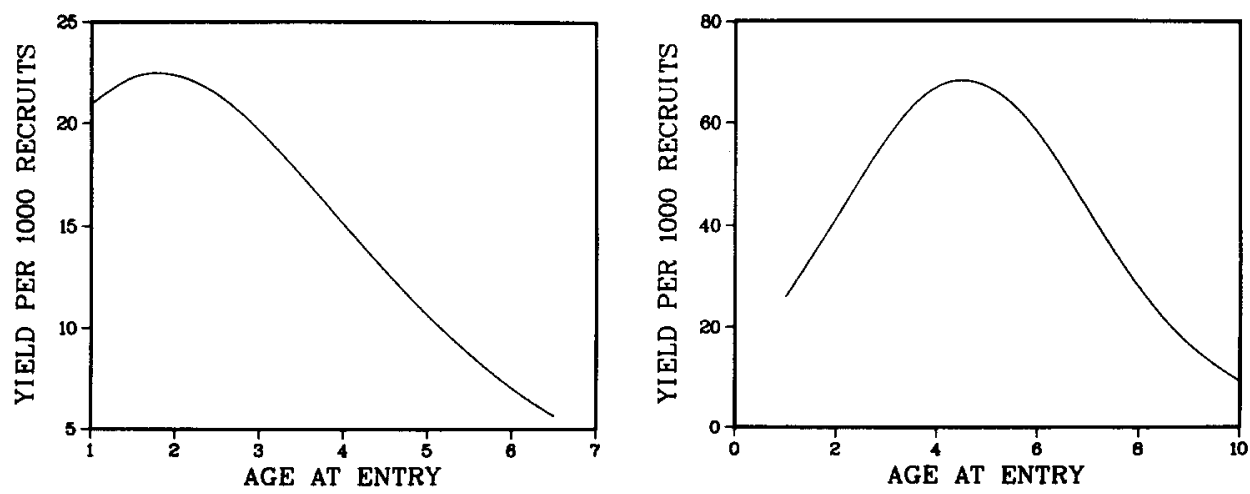

Fig. 5. Yield per 1000 recruits as a function of age at entry, $x_{c}$, assuming knife-edge recruitment.

Fig. 6. Yield per 1000 recruits as a function of mean selection age, $k$, using the adjusted catch curve. 


\section{DISCUSSION}

Gill nets are efficient gear for catching widely dispersed fish and can be fished from small boats with small investment in manpower and gear (Hamley 1975). Gill nets are selective, however, and this selectivity may bias estimates of population size, mortality, growth, and recruitment. Selection curves are usually unimodal but bimodal and multimodal curves have been reported (Hamley and Regier, 1973; Hamley, 1975). Fish are usually caught by the head, but they may be wedged in the mesh or tangled by teeth, fin rays, or other body projections. These modes of capture can produce complex selection curves (Hamley and Regier, 1973; Hamley, 1975). Several probability distributions have been applied to describe gill net selection (Hamley, 1975). The normal distribution is the most commonly applied distribution (Hamley, 1975). These distributions have been applied to estimate age-specific selectivities which are used to adjust the observed catch data for gear selection before applying the data to estimate population parameters. In this study the selectivity curve was combined with the mortality equation to give a catch curve adjusted for gill net selection. This approach is especially useful for simulation of the impact of changes in mesh size and fishing effort on age structure, age-specific fishing mortality, and yield.

The logistic distribution was applied to describe the selection curve because it is similar in shape to the normal distribution and it can be integrated in closed form. The adjusted catch curve incorporates the observed continuous change in vulnerability of an exploited stock with age. The adjusted catch curve can be used to calculate age-specific fishing mortality coefficients and to examine yield per recruit as a function of both mean selection age and fishing mortality.

\section{ACKNOWLEDGEMENT}

I thank Dr. G.L. Kesteven and the referees for many helpful suggestions that have much improved the style and clarity of this paper.

\section{REFERENCES}

Beverton, R.J.H. and Holt, S.J., 1957. On the dynamics of exploited fish populations. U.K. Min. Agric. Fish., Fish. Invest. (Ser. 2), No. 19, 533 pp.

Brown, E.H., 1979. Population dynamics and interagency management of chubs (Coregonus spp.) in Lake Michigan. Mimeo. Rep. Great Lakes Fishery Laboratory, Ann Arbor, MI. MI, 38 pp.

Gulland, J.A., 1969. Manual of Methods for Fish Stock Assessment. Part I. Fish Population Analy sis. F AO Manuals in Fisheries Science, No. 4, 154 pp.

Hamley, J.M., 1975. Review of gillnet selectivity. J. Fish. Res. Board Can., 32: 1943-1969.

Hamley, J.M. and Regier, H.A., 1973. Direct estimates of gillnet selectivity to walleye (Stitzostedion vitreum). J. Fish. Res. Board Can., 30: 817-830. 
Holt, S.J., 1963. A method of determining gear selectivity and its application. Int. Comm. Northwest Atl. Fish. Spec. Bull., 5: 106-115.

Jensen, A.L., 1978. Assessment of the lake trout fishery in Lake Superior: 1929-1950. Trans. Am. Fish. Soc., 107: 543-549.

Jensen, A.L., 1981. Adjusting fishery catch curves for trawl selection using the logistic distribution. J. Cons., Cons. Int. Explor. Mer, 40: 19-23.

Johnson, N.L. and Koltz, S., 1970. Distributions in Statistics: Continuous Univariate Distributions - 2. John Wiley \& Sons, New York, NY, 306 pp.

Regier, H.A. and Robson, D.S., 1966. Selectivity of gill nets, especially to lake whitefish. J. Fish. Res. Board Can., 23: 423-454. 